\title{
Filariasis in Bone Marrow Aspiration of a Child with Pyrexia of Unknown Origin - A Case Report
}

\author{
Dr Sangeeta Sharma ${ }^{1}$, Dr Ujwala Maheshwari ${ }^{1}$, Dr Pooja Gajaria ${ }^{1}$, \\ Dr Reeta Dhar ${ }^{1}$, Dr Sudhir N Kadam ${ }^{2}$ \\ 1 (Department of Pathology, MGM Medical College and Hospital, Navi Mumbai, Maharashtra, India) \\ ${ }^{2}$ (Medical Director, MGM Medical College and Hospital, Navi Mumbai, Maharashtra, India)
}

\begin{abstract}
A variety of haemoparasites infecting humans occur in the tropical and subtropical regions of the world. Filariasis is one of them that usually manifests as hydrocoele, elephantiasis, lymphadenopathy and fever. The microfilaria causing these symptoms can usually be demonstrated in the peripheral circulation or get lodged into one of the organs and are discovered on fine needle aspiration samples. However, occurrence of microfilaria in the bone marrow is a rare presentation. We hereby report one such case of a paediatric patient who presented as a case of pyrexia of unknown origin, anaemia and raised creatinine levels.
\end{abstract}

Keywords: Anaemia, Bone marrow, Creatinine, Filariasis, Pyrexia, Wuchereria bancrofti

\section{INTRODUCTION}

Out of the 8 species in the world, Filariasis in India is caused by Wuchereria bancrofti or Brugia malayi, both of which are transmitted through Culex mosquitoes. Filariasis is usually diagnosed by demonstrating circulating microfilaria in the peripheral blood of the patient collected between 12.00 AM and 4.00 AM. During circulation these microfilaria can get lodged in various organs and can be demonstrated in Fine Needle Aspiration (FNA) samples from the thyroid, breast, subcutaneous nodules or other organs, or in tissue samples such as bronchial washings.[1-7] Detection of microfilaria in bone marrow aspirates is a rare finding. A few cases, which have been reported, were usually associated with pancytopaenia, [8-12] and one case was incidentally discovered in a patient with acute lymphoid leukaemia. [13]

\section{CASE REPORT}

A 12 year old girl presented to the Hospital with high grade intermittent fever with spikes for 4 days, along with by 2-3 episodes of vomiting per day, decreased oral intake, and abdominal pain. There was no history of haematuria, dysuria or any other past illness. On examination, the patient was febrile, poorly built, and showed pallor.

There was no evidence of icterus, clubbing, bony tenderness, lymphadenopathy or organomegaly. On admission her Haemoglobin was $6.7 \mathrm{gm} / \mathrm{dl}$, Mean Corpuscular Volume (MCV) $89.3 \mathrm{fl}$, Mean Corpuscular Haemoglobin(MCH) $27.7 \mathrm{pg}$, and Mean Corpuscular Haemoglobin concentration (MCHC) 31.0\%. The Peripheral Blood Smear revealed predominantly normocytic normochromic RBCs along with few microcytes and pencil cells. The Total Leukocyte Count was raised to 24,200 cells per cumm, with a Differential Count revealing Eosinophilia (P-74, L-16, E-10). The Absolute Eosinophil Count was 2420 cells per cumm . Thrombocytopenia was observed with the Platelet Count being 90,000 per cumm. Serum Creatinine was raised to 4.1. Tests for detection of Malarial Parasite, Dengue, Leptospira and Typhoid were negative.

With the cause of fever unknown and anaemia not responding to treatment, a bone marrow aspiration was performed from the anterior superior iliac spine. The smears, stained with Leishman's stain, were normocellular with a M:E ratio of 6:1. Erythroid series was predominantly normoblastic with a few micronormoblasts. eosinophils and its precursors were mildly increased to $15 \%$ along with increased plasma cells (12\%) and iron laden macrophages (Fig. 1). Megakaryocytes were morphologically normal and even in distribution.

One of the smears revealed a sheathed organism resembling microfilaria (Fig. 2). Its nuclei did not extend till the tail end and therefore,this organism was morphologically typed as Wuchereria bancrofti. Subsequently, a midnight peripheral smear examination revealed multiple such microfilaria. The patient was treated with Diethylcarbamzine and Albendazole, and given two units of packed cells. She showed improvement with her Haemoglobin rising to $8.9 \mathrm{~g} / \mathrm{dl}$ and Platelets increasing to 1.40 lakhs per cumm. Serum Creatinine levels also returned to normal. 


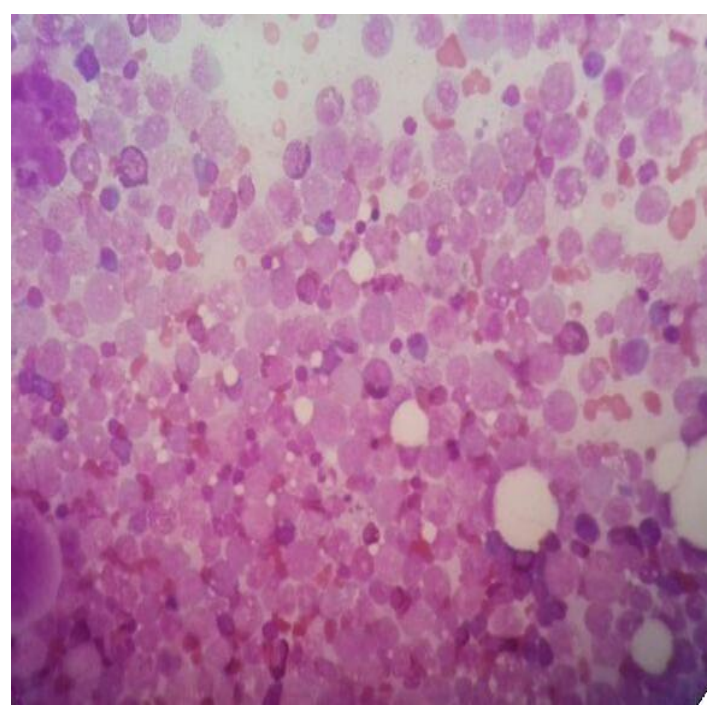

Figure 1: Bone Marrow Aspiration smear (stained with Leishman's stain) showing increased Eosinophils and its precursors, along with increased Plasma Cells, and iron laden Macrophages

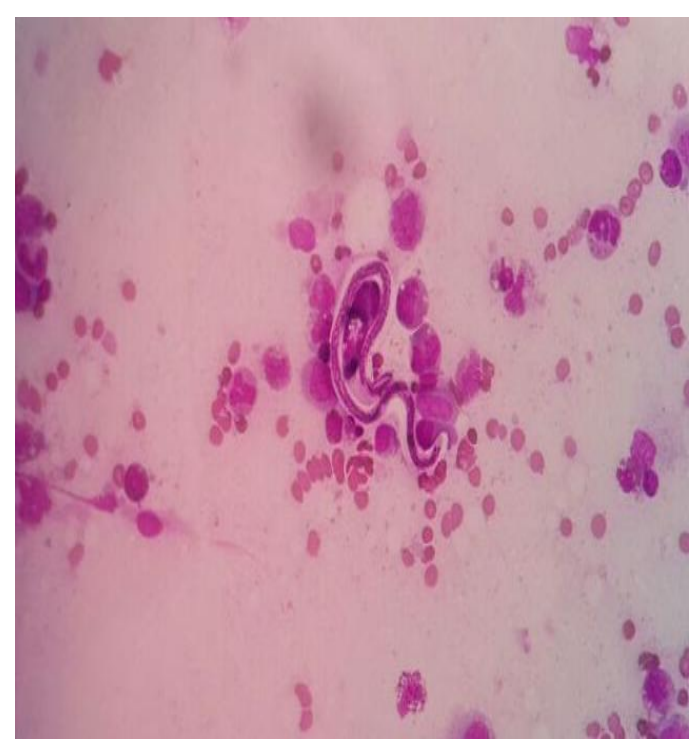

Figure 2: Bone Marrow Aspiration smear revealing a sheathed microfilaria with nuclei not extending till the tail end

\section{DISCUSSION}

Wuchereria bancrofti is the cause of Filariasis in $98 \%$ of the cases. It was first demonstrated in the hydrocoele fluid of a man by Demarcuay in 1863.[14] Humans act as a definitive host and mosquitoes are the intermediate host. The larvae enter through the skin, reach lymphatic channels and settle down in the lymph nodes. They mature and produce new larvae in 15-18 months. These then enter through the thoracic duct into the venous system and finally into the peripheral circulation. While circulating, these get lodged into a variety of organs and are picked up in Fine Needle Aspiration smears from the thyroid, breast, subcutaneous nodules or other organs, or in tissue samples such as bronchial washings.[1-7] Their ability to cross the vessel wall allows them to settle in the bone marrow also.

In our patient, Filariasis was not suspected clinically due to the lack of a typical clinical picture. Bone marrow aspirate to evaluate pyrexia of unknown origin and anaemia refractory to treatment, revealed the incidental finding of microfilaria. This finding prompted the collection of a midnight blood sample and the subsequent finding of microfilaria in the peripheral smear.

According to a study conducted by J. Langhammer et al. [15], renal failure can occur due to tubular damage, even in asymptomatic microfilaraemia, which explains the high creatinine levels in our patient. In symptomatic patients, there is glomerular damage occurring as a result of circulating immune complexes, which usually manifests as proteinuria and/or haematuria. 
Umashankar et al. [8] have reported microfilaria in bone marrow aspirates with megaloblastic anaemia.Sharma et al. [9] described six cases of microfilaria in bone marrow aspirates, among which five had bone marrow hypoplasia or aplasia. Debanjali et al. [10] also reported a case of pancytopenia with microfilaria in the bone marrow. A single case report of microfilaria, in association with acute lymphoid leukaemia, has been reported by Arundhati et al. [13]. But all these cases lacked peripheral blood Eosinophilia.

Our case was unique in having bicytopaenia with raised Total Leukocyte Count and peripheral blood Eosinophilia along with features of acute renal failure presenting with high creatinine levels which later returned to normal, after successful treatment.

\section{CONCLUSION}

In endemic areas, a thorough work up of patients to rule out Filariasis is a must, especially in patients presenting with pyrexia of unknown origin and anaemia along with raised creatinine levels.

\section{ACKNOWLEDGEMENTS}

Our special gratitude to the Department of Paediatrics, MGM Medical College, and Hospital, Navi Mumbai.

\section{REFERENCES}

[1]. Yenkeshwar PN, Kumbhalkar DT, Bobhate SK. Microfilariae in fine needle aspirates: a report of 22 cases. Indian J Pathol Microbiol 2006;49:365-69.

[2]. Kishore B, Khare P, Gupta RJ, Bisht SP. The microfilariae of Wuchere- ria bancrofti in cytologic smears: a report of 5 cases with unusual presentations. Acta Cytol 2008;52:710-12.

[3]. Gupta S, Gupta R, Bansal B, Singh S, Gupta K, Kudesia M. The significance of the incidental detection of filariasis on aspiration smears: a case series. Diagn Cytopathol 2010;38:517-20.

[4]. Anupindi L, Sahoo R, Rao RV, Verghese G, Rao PV. Microfilariae in the bronchial brushing cytology of symptomatic pulmonary lesions. A report of two cases. Acta Cytol 1993;37:397-99.

[5]. Srikanth S. Microfi laria in thyroid aspirate-An unusual finding. Thyroid Research and Practice | January-April $2014 \mid$ Vol $11 \mid$ Issue 1:26-8.

[6]. Col R Ravikumar,Col KK Sen, Surg Lt Cdr SN Singh,Surg Capt N Chawla, SR Choudhary, Maj D Singh. Filarial Dance in a Breast LumpMJAFI 2010; $66: 193-195$

[7]. Kanade. U. S, Dantkade. S. S, Chaware. S. A, Narkhede. R. R, Gadhekar. M. S.Microfilaria in preauricular subcutaneous nodule on cytology - an unusual presentation. Journal of Evolution of Medical and Dental Sciences/Volume1/Issue4/October - 2012 Page 382386

[8]. Umashankar T, PJ Yaranal Microfilariae in Bone Marrow Aspirates: Report of a Case. Journal of Clinical and Diagnostic Research, 2012 September, Vol-6(7): 1294-1295

[9]. Sharma S, Rawat A, Chowhan A. Microfilariae in bone marrow aspiration smears; their correlation with marrow hypoplasia: a report of six cases. Indian J Pathol Microbiol 2006;49:566-68

[10]. Debanjali Sinha, Sumantro Mondal, Tony Ete, Arijit Nag, Kaushik Bhar, Sattik Siddhanta, Jyotirmoy Pal, Alakendu Ghosh filariasis presenting with pancytopenia diagnosed by microfilaria in bone marrow aspirate - report of a rare entity from India. International Journal of Medical Science and Public Health | 2014 | Vol 3 | Issue 5; 638-639

[11]. Shailesh S Vartak, Urmi S Chakravarty-Vartak, Sandip Parate, Sona Balan Microfilaria in A Bone Marrow Aspirate. Bombay Hospital Journal, Vol. 50, No. 1, 2008; 90-91

[12]. Sasidharan PK, Geetha P, Shaan M, Sreejith R and Aslam M Microfilaria Causing Bone Marrow Failure .Journal of Case Reports and Studies Volume 2 Issue 1

[13]. Arundhati, Ashok Kumar, Rakesh Kumar Acute Lymphoblastic Leukaemia with Microfilaria: A Rare Coincidence in Bone Marrow Aspirate.Indian J Hematol Blood Transfus (Apr-June 2011) 27(2):111-112

[14]. Parasitology protozoology and helminthology in relation to clinical medicine $13^{\text {th }}$ edition K.D.Chatterjee

[15]. J. Langharnrner, H. -W. Birkl and H. Zahner Renal disease in lymphatic filariasis: evidence for tubular and glomerular disorders at various stages of the infection.Tropical Medicine and International Health Volume Z NO 9 PP 875-884 September 1997. 